\title{
MEETING SUMMARIES
}

\section{NEXT-GENERATION NUMERICAL WEATHER PREDICTION Bridging Parameterization, Explicit Clouds, and Large Eddies}

\author{
By SONG-You Hong AND Jimy DUdHIA
}

$\int$ ix years after the second international workshop on the physical parameterizations in numerical weather prediction (NWP) models in 2004 (Lee and Hong 2005), attendees at the third workshop on next-generation NWP models met to discuss progress in high-resolution NWP modeling and share ideas about future challenges. The main theme of the meeting was "The Cloud-Resolving Modeling Approach and Beyond." As of 2010, convection-permitting and cloud-resolving scale modeling has become practically feasible, along with the successful usage of largeeddy simulation (LES) in developing subgrid-scale parameterizations for these models. Many national hydrometeorological centers are now running models in the $2-5-\mathrm{km}$ grid-size range, and will be increasing resolution at a steady rate such that several centers may be at around $1 \mathrm{~km}$ in $5 \mathrm{yr}$. The main topic of the workshop focused on future problems in physics as NWP models go to finer scales where there are

AFFILIATIONS: Hong-Department of Atmospheric Sciences, Yonsei University, Seoul, South Korea; DUDHIA—MMM Division, NCAR, Boulder, Colorado

CORRESPONDING AUTHOR: Song-You Hong, Department of Atmospheric Sciences, Yonsei University, Seoul 120-749, South Korea

E-mail: shong@yonsei.ac.kr

DOI:10.II75/20IIBAMS3224.I

In final form 24 August 2011

(C)2012 American Meteorological Society

\section{THE THIRD INTERNATIONAL WORKSHOP ON NEXT-GENERATION NWP MODELS}

What: Scientists from Korea, Japan, France, England, Finland, and the United States met to discuss recent developments in the parameterizations of physical processes in next-generation, highresolution numerical weather prediction (NWP) models (http://nml.yonsei.ac.kr/20100829/).

WHEN: 30 August-I September 2010

Where: Seoguipo, Jeju, South Korea

"gray zones" in which the explicit model dynamics are almost capable of resolving features that were parameterized at coarser scales. The following questions were posed for the workshop:

- At what scales do the one-dimensional (1D) vertical mixing planetary boundary layer (PBL) schemes, which are usually separated from horizontal mixing, work?

- When do they need to be replaced with threedimensional (3D) large-eddy-simulating turbulence models?

- At what scales are models considered to be convection permitting?

- When is a cumulus parameterization scheme (CPS) needed?

- When is a separate shallow convective scheme needed? 
- What are the microphysics challenges at high resolution?

- How can we handle the gray-zone issues in convection and boundary layer physics?

Forty-five presentations were given, which covered the current status of high-resolution NWP and cloud-resolving models, progress with physical parameterization, and the application of LES to the development of subgrid-scale processes.

\section{CURRENT STATUS OF NWP MODELS} AND SUBGRID-SCALE PHYSICS. The workshop began by demonstrating the current capability of high-resolution NWP systems, including the North American Mesoscale (NAM; United States), Application of Research to Operations at Mesoscale (AROME; France), Unified Model (UM; United Kingdom and also used in Korea), HighResolution Limited-Area Model (HIRLAM)-Aire Limitée Adaptation Dynamique Développement International (ALADIN) Regional/Mesoscale Operational NWP in Europe (HARMONIE; Europe), nonhydrostatic model (NHM; Japan), and experimental severe storm and hurricane forecasts with the Weather Research and Forecasting model (WRF) at the National Center for Atmospheric Research (NCAR). Several presentations on real-time forecasts demonstrated that the NWP models at grid sizes of $1-5 \mathrm{~km}$ provide reliable information for weather forecasts, in particular, precipitation. At this resolution, some presentations from operational centers showed the importance of turbulence mixing length and horizontal diffusion in improving boundary layer clouds (stratocumulus, shallow cumulus, and fog). While the removal of the CPS physics process is widely favored in the research community, some of operational model evaluations showed that its inclusion is beneficial in alleviating spurious rainfall peaks. There was a smaller sensitivity to the PBL scheme choice than to the cloud microphysics choice in the NCAR severe storm study.

Research activities on multiscale modeling were presented for various models. These included results from the German icosahedral-hexagonal gridpoint global model (GME) on medium-range forecasts; the Japanese Nonhydrostatic Icosahedral Atmospheric Model (NICAM), with a global cloud-resolving scale simulation; the Korean Global/Regional Integrated Model system (GRIMs), introducing its physics development test beds; the National Aeronautics and Space Administration (NASA) Goddard multiscale modeling system, showing convective system simulations; the Colorado State University (CSU)-University of California, Los Angeles (UCLA) quasi-3D (Q3D) multiscale modeling framework (MMF), with efficient superparameterization; and the National Taiwan University (NTU)-Purdue University nonhydrostatic model compared with the WRF for finescale complex topography.

Presentations discussed the role of CPSs in the gray zone and suggested revisions for a smooth transition from convection-permitting to cloud-resolving scales. Several presentations showed the importance of chemistry processes to better simulate clouds and precipitation. The interaction of PBL turbulence parameterizations with other physics was shown in weather forecasts and climate simulation, especially sensitivities between PBL schemes regarding lowcloud formation, convective initiation, convectively modified boundary layers, and upper-level gravity wave drag. The significance of convective sources of momentum and gravity waves on large-scale circulations in the stratosphere was also shown. The gridsize dependence of the partitioning of subgrid- and grid-scale energy in the PBL was quantified in one parameterization intercomparison.

There were three breakout discussion groups in the focus areas of clouds, boundary layers, and atmospheric chemistry, leading to talking points for a final plenary discussion on the above-listed workshop topic questions; this is summarized here.

\section{CHALLENGES IN PBL PARAMETERIZA- TIONS TO LARGE-EDDY RESOLVING} SCALES. It was recognized that $1 \mathrm{D}$ PBL schemes are adequate at grid sizes less than $1 \mathrm{~km}$, and probably $500 \mathrm{~m}$, which means that it may be a decade before the PBL gray zone issues need to be addressed in national forecast models. This time scale is governed by computing limitations that make 1-km real-time forecasts costly to do at reasonable speed with current computers. There was also a discussion about the use of 3D subgrid mixing schemes near 1-km grid sizes (as used for many years in cloud-resolving research models), but they are not designed for cases of strong surface fluxes and boundary layer development.

At grid scales nearer to $100 \mathrm{~m}$, it is considered that vertical eddy mass fluxes will be resolved sufficiently by the dynamics so that the nonlocal or so-called counter-gradient subgrid mixing effects of PBL schemes will no longer be needed, and all of the mixing can be considered local as with LES parameterizations. LES parameterizations work well in the inertial subrange, but even their assumptions break down near the surface and in stable conditions 
as more of the energy-containing eddies become subgrid scale.

However, while dry vertical mixing may be adequately handled down to $1 \mathrm{~km}$ with current physics schemes, challenges include shallow convection and the boundary layer response to resolved deep-cloud downdrafts and subsidence around clouds, and, conversely, the impact of the boundary layer on resolved deep convection and moist processes in general. These also include how to handle fractional cloud coverage, both regarding their mass fluxes and radiative effects. Also, PBL schemes that have shallow convective components are still in need of further evaluation and development, which is achieved to some extent by the practice of using LES-scale models as "truth."

\section{CHALLENGES IN CUMULUS PARAM-} ETERIZATION TO CLOUD-PERMITTING

SCALES. With a variety of cumulus schemesfrom mass flux to adjustment to moisture convergence type-some operational physics suites do better at $5 \mathrm{~km}$ without the cumulus scheme, while others do worse when using it at the same resolution. There are situations where employing a convective scheme is better because it triggers deep clouds more quickly than the microphysics and dynamics can by themselves, which also mitigates a tendency toward a high-intensity bias that might occur without it [e.g., the National Centers for Environmental Prediction (NCEP)'s Regional Spectral Model (RSM), and Japanese Meteorological Agency's (JMA) NHM]. In contrast, an adjustment-type scheme produced unrealistically smooth structures at $4 \mathrm{~km}$ in the NCEP regional Nonhydrostatic Mesoscale Model (NMM) system, and the model worked better without it. The new ALADIN cumulus scheme (Gerard et al. 2009) was designed to work reasonably well at $3-8 \mathrm{~km}$ by allowing subgrid prognostic updraft effects that closely interact with the microphysics. Cumulus schemes for these scales need to relax the scale separation assumption that subsidence occurs in the same grid column as the updraft, as with the Grell 3D cumulus scheme in the WRF that spreads subsidence to neighboring columns. This was presented at the workshop, although traditional schemes still seem to work regardless of making the single-column assumption. Ideally, gray zone cumulus schemes should "turn themselves off" as the resolved scale takes over and vice versa as the grid size gets coarser. It was generally agreed that no deep cumulus scheme is justified at $1-2.5 \mathrm{~km}$, but a shallow convective parameterization is needed either independently of the deep cumulus scheme or as part of the PBL parameterization.

Several issues make it difficult to determine a clear grid size where convection permitting can be assumed adequate. These issues include the dynamics and numerical techniques of the underlying model, for example, Eulerian, semi-Lagrangian, diffusiveness, and the type of convection to be simulated. For example, severe storms in the United States as well as tropical cyclones seem to be well captured with 3-4-km grid sizes, possibly because of their large mass fluxes and mesoscale self-organization; however, it is not clear that isolated convection with narrower and weaker updrafts developing from weak forcing would be adequately resolved.

Several research efforts are using or planning to use large datasets from cloud-resolving models to continue the development of cumulus parameterizations, and the possibility of using LES models was discussed. The subgrid convection problems at GCM gridbox sizes are different from those in NWP regional grids because the former needs to include some organization effects, while the latter probably includes individual convective cloud effects. As the NWP model resolution shrinks below $3 \mathrm{~km}$, cumulus parameterization will become obsolete, but it was recognized that some parameterization will still be needed for coarser large-area domains that will continue to be employed for data assimilation and ensembles. Nesting LES models within NWP models for local high-resolution studies (e.g., urban areas and wind farms) was discussed, but it was agreed that for LES models to behave realistically, their upstream boundary needs to be far enough from the area of interest for eddies to develop.

\section{CHALLENGES IN MICROPHYSICS AND CHEMISTRY. For microphysics, the main ques-} tion for forecast models is whether to go to doublemoment schemes that predict number concentrations and have more flexibility to properly distinguish the effects of aerosols for cloud and ice nucleation. This would make sense in forecast systems that use chemical data assimilation, which are likely to be developed more in the future. It was also suggested that mixed-phase growth into hail or graupel is often treated too simply, and there are now several schemes that better handle riming as a gradual, rather than a discrete, process.

Regarding atmospheric chemistry, it was recognized that mass flux-type cumulus schemes are capable of chemical transport, and models including this process can have their schemes evaluated with 
chemical tracer observations, as well as meteorological ones. Boundary layer and cumulus schemes should be developed keeping in mind mixing multiple chemical tracers for generality. Levels of complexity of chemistry exist in models, from simpler aerosol or dust only to gas phase, aqueous phase, and sectional (bin) models. For their initial application to real-time NWP, probably only the simpler chemistry modules are computationally feasible. Several NWP centers are considering aerosols initially for visibility and dust prediction, and it was recommended that aerosol radiative and microphysical effects, as well as air quality applications, should become integral parts of future NWP systems.

FURTHER ISSUES FOR HIGH-RESOLUTION MODELING. Data assimilation in relation to high-resolution models was briefly addressed. Current systems often do data assimilation at lower resolution than the cloud-resolving models, and there would be a spinup delay for finescale structures unless the first guess from the finescale model is cycled (as at the Met Office, e.g.). Data at cloud-resolving scales remain a challenge because of the poor match between the data and model resolutions that do not constrain the model well enough. It was recognized that at finescales, deterministic forecasts are not likely to give as useful guidance as high-resolution ensembles, which give a measure of uncertainties, especially with convective systems.
A fourth workshop on this topic is expected within $5 \mathrm{yr}$. Abstracts and presentation materials from this workshop, as well as a list of presenters, are available online (http://nml.yonsei.ac.kr/20100829/).

ACKNOWLEDGMENTS. The authors would like to express thanks to Masao Kanamitsu for providing ideas on the main theme of the workshop in the early stage of organization. This work was supported by the Basic Science Research Program through the National Research Foundation of Korea (NRF), funded by the Ministry of Education, Science and Technology and by the Korea Meteorological Administration Research and Development Program under Grant RACS_2011-2023. The authors are grateful to Dong-Kyou Lee, Tae-Young Lee, Yign Noh, Hye-Yeong Chun, Rokjin Park, Sang-Wook Yeh, Jong-Seong Kug, Dong-Eon Chang, Young-Hwa Byun, Cheon-Ho Cho, Woo-Jin Lee, and Won-Tae Kwon for their financial support and encouragement.

\section{REFERENCES}

Gerard, L., J.-M. Piriou, R. Brožková, J.-F. Geleyn, and D. Banciu, 2009: Cloud and precipitation parameterization in a meso-gamma-scale operational weather prediction model. Mon. Wea. Rev., 137, 3960-3977.

Lee, T.-Y., and S.-Y. Hong, 2005: Physical parameterization in next-generation NWP models. Bull. Amer. Meteor. Soc., 86, 1615-1618. 